\title{
MExS A Fuzzy Rule Based Medical Expert System To Diagnose The Diseases
}

\author{
Sajan Seth \\ College Of Computer Science Bhai Maha Singh College of Engg \& Tech., India \\ sajan.sajan570@gmail.com
}

\begin{abstract}
This article describes the development of medical expert system for diagnosis of human diseases [MExS]. MExS can be used to perform some statistical evaluations on patient's symptoms which we find in our daily life, to detect the particular disease. It is a decision support system based on the symptoms. We describe a new method to creating weighted fuzzy set rule to deal with the medical diagnosing problems from the training data. It is a causal rule (whose "IF" part truly causes the "THEN" part to happen as an effect) based medical diagnosis expert system. The researchers are try to advance to develop software that will assist the doctors, physician or a normal human being in making right decision without any consultation with the specialist.

The aim of this paper is to develop a single expert which deals with the diseases of liver, kidney, ENT, MD, blood, nervous system, eye, and skin. This expert system is a collection of different specialists related to human into a single unit to predict the human diseases.
\end{abstract}

Keywords: - Diagnosis 25 human diseases, Fuzzy logic, Medical record of patients, Suggest specialist.

\section{INTRODUCTION}

In a medical field diagnosis is a methodology for identification or recognition of a disease based on the some signs and symptoms that appears. One of the major issues in the diagnosis of disease is risk stratification. This MExS is use for simplifying the task of physician. MExS checks the initial stage of patient and find out the result on basis of sign and symptoms and tell the corresponding diseases and also suggest the corresponding specialist. In medical application it is widely used to predict the human disorder of body like, disorder of liver (Hepatitis A, Hepatitis B, Cirrhosis), disorder of blood(Anemia, Leukemia, Hodgkin's Disease), diseases of ear nose throat(External otitis, Serous otitis, Acute rhinitis, Acute Follicular, Acute bronchitis, Acute Pharyngitis), disorder of nerve system(Strokes, Migraine, Meningitis, subarachnoid hemorrhage), disorder of kidney(Polycystic, Pyelonephrities, Nephritic Syndrome), disorder of Heart(Conginential heart Disease, Pneumonia, Asthma, Angina, Acute Rheumatic heart disease, Myocardial infaction) and some common diseases of MD (Common cold, influenza, hypertension, rubella, rabies, typhoid, malaria). Identification of the parameter is the most important part to take into the consideration. This medical expert system starts with asking some symptoms to the patient, if the system is able find the disease then it provide the name that particular disease on the basis of available cumulative information and refer to a appropriate specialist. If the system is not capable of identifying the disease on basis of symptom, it will ask some sign and symptoms to the patients. MExS not only simplifies task of the doctors but it will also helps the patients by providing initial medicines for small diseases in emergency.

Research has shown [1] that when medical experts approach the problem of diagnosing an illness, based on limited findings provided in the consultation they quickly generate a small number of disease hypotheses, and then, they seek to confirm or refute these hypotheses with further specialized examination and testing.

\section{DEVELOPMENT PROCESS OF MEDICAL DIAGNOSIS SYSTEM}

The medical diagnosis system process is classified into four categories:

1. Collection of Symptoms Data: The most important information about the patient's medical record present in the hospitals and the symptoms that are present is gathered by the medical experts or physician. This information considered as the 'subjective information'. This information is further helpful for diagnosis of particular disease.

2. Interaction with Expert: The next step is to interaction with the expert or specialist. The expert deal with the daily problems related with disorder of human body parts. It will tell you the more details of the common diseases which found in our daily life.

3. Categorization of Diseases and Symptoms: After interacting with the expert, MExS generates the list of diagnosis that describes the sign and symptom of patient. This phase show the categorization of the sign and symptoms. Multiple diseases share the individual symptoms. Due to The presence of single symptom the disease can change. For assisting the medical diagnosing system for Human disease diagnosing, to building a knowledgebase environment is a complex task because the specific importance of all the data of medical. One 
another is reason is that the interpretations that are given by different doctors to the patients. So categorization is important. Consider an example, cough is not a vital symptom, but from this symptom multiple diseases can occur like TB acute bronchitis, Hodgkin's disease, common cold, influenza. These diseases are from different experts.

4. Rule Formulation: In this phase we implement the fuzzy rules of the sign and symptom and the diseases. Here we create weighted fuzzy set rule to deal with the medical diagnosing problems from the training data. It is a causal rule (whose IF part truly causes the THEN part to happen as an effect) based medical diagnosis expert system. If the patient is suffering from the symptoms pallor of skin is severe, weakness is high, giddiness is high, heart rate is also severe, and then the patient is sure suffering from the disease anemia. This disease is related to the disorder of blood.

5. Expert Review of Rule Base: in this phase, we evaluate the rule viewer of the rule base. It gives to the result. On the basis of these symptoms particular disease is evaluated and refers the patient to a particular specialist. The disease is evaluated on the basis of knowledge of expert. Suppose a patient is suffering from fever with shivering attack. There are two possible diseases that are possible based on their weighted. If the weight age of the symptom is high the patient is suffering from the malaria. If the weightage is low then there is possibility that the patient is suffering from the Pneumonia.

6. Finalization of Rule Base: In this phase the final output is evaluated on the basis of the sign and symptom. For example if the patient is suffering from the symptom loss of sight, opacity of lens and blurring of vision then patient is suffering from the disease of eyes and he/she would refer to Ophthalmologist (Eye specialist) for their treatment.

7. Designing of User Interface for Interaction: The last phase of the development process is designing of graphical user interface. This GUI will help to the user to interact with this expert to find the particular disease. This system takes the symptom as a input and gives the output as a disease. After finding the output it suggests the specialist for the treatment. This GUI will help to the user to interact with this expert to find the particular disease. This system takes the symptom as an input and gives the output as a disease. Mainly the system has following module:

\section{Knowledge Base:}

the vital symptoms of patients are added to this system. Weights are assigned to individual symptom that is stored into the rule base and the symptoms are fuzzified.

\section{GUI Interface:}

This module provides the graphical user interface that shows the patient information and provides the vital sign and symptoms that may occur. This system provides the interaction between the user and the expert system.

\section{Inference Engine:}

In this module where the defuzzification of the system is done.

So many problems in the diagnostic medical expert system represented at degrees of abstraction to be solved. Consider an example, a patient tell a doctor that he is suffering from the general malaise. The doctor gives simply suggest taking a rest and if this treatment is failed, then he/she can have to deal with more detailed information of the symptom i.e. general malaise from which particular disease can occur. And also able suggests the corresponding specialist or expert.

The development process of medical diagnosis expert system is shown in fig 1 .

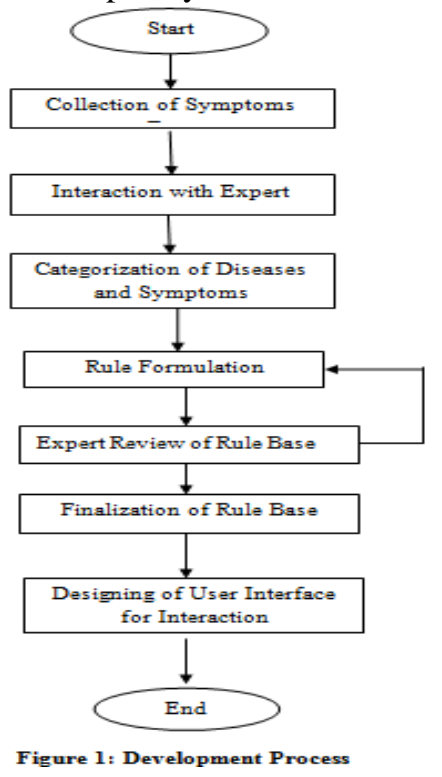




\section{METHODOLOGY}

Fuzzy Logic was developed by Zahed in 1965. The reasoning of the human which is imprecise needed to be handled efficiently. Fuzzy logic gives the kind of logic which became more important day to day lives. Fuzzy is a multi valued logic deal with the reasoning. Fuzzy logic applied in the different fields from a small embedded system to large controller. In medical applications it is widely use include Predicting the disorder of liver functioning, disorder of eye, disorder of kidney, disorder of the nerve system, disorder of blood, disorder of ear nose throat(ENT). There are so many papers which deal with the fuzzy logic in medical applications. These papers deal within the particular field.

Identification of parameters: - this is the most important part to take into the consideration. The symptoms play the significant role in cause of the disease. In this paper we find the different diseases that are associated with symptoms of each specialist.

Choice of membership function: - the efficiency of system is determine by the choice of membership function that is used for every input and output parameters. There are various symptoms that do not have a particular values and the membership function are chosen for each of the parameter which is used. These membership values are taken on the basis of intuition method. Besides the number of membership function used there are some another factors need to be take account and these factors are the conjunction, disjunction, aggregation, type of parameters and the range of the parameters. The characteristic of each fuzzy variable is represented using triangular (for the Outputs) and the trapezoidal (for the input variables such as symptoms). In this paper, the fuzzy set represented by a number of membership function. In this paper we considering the diseases of various specialist and these specialists are, Gastroenterologist, Ophthalmologist, Nephrologists, Hematologist, Cardiologist, Neurologist, Dermatologist, MD and ENT. This expert system deals with 45 diseases of the different specialist.6200 rules are generated. The single example is listed below:

In a medical field diagnosis is a methodology for identification or recognition of a disease based on the some signs and symptoms that appears. In medical application it is widely used to predict the human disorder of body like, disorder of liver (Hepatitis A, Hepatitis B, Cirrhosis), disorder of blood(Anemia, Leukemia, Hodgkin's Disease), diseases of ear nose throat(External otitis, Serous otitis, Acute rhinitis, Acute Follicular, Acute bronchitis, Acute Pharyngitis), disorder of nerve system(Strokes, Migraine, Meningitis, subarachnoid hemorrhage), disorder of kidney(Polycystic, Pyelonephrities, Nephritic Syndrome), disorder of Heart(Conginential heart Disease, Pneumonia, Asthma, Angina, Acute Rheumatic heart disease, Myocardial infaction) and some common diseases of MD (Common cold, influenza, hypertension, rubella, rabies, typhoid, malaria). Identification of the parameter is the most important part to take into the consideration. The input and output parameters are the symptoms and diseases. It takes the symptom as a input and it produce the output as the disease. The efficiency of the system is determined by the choice of membership function that is used for individual input and the output. There are various symptoms that do not have a particular values and the membership function are chosen for each of the parameter which is used. These membership values are taken on the basis of intuition method. The characteristic of each fuzzy variable is represented using triangular (for the Outputs) and the trapezoidal (for the input variables such as symptoms). Gastroenterologist is one of the largest organs in the human body. It placed the right half of the diaphragm. It has weights about $1.5 \mathrm{~kg}$. The normal liver consists of masses of liver cells. Running between the masses of liver cells are tinny bile channels. The liver plays an important role in human body. It receives the blood from vein. Blood passes through the liver before entering the circulation. Disorder of Liver contains Hepatitis A which is spread by the faeco-oral route with period 4-5 weeks. It usually mild infection of children which goes unnoticed. Hepatitis B is a very widespread infection with period 3-6 months. It is transmitted by blood, syringes, tattooing. It is probable that it can also be transmitted by kissing or sexual intercourse. Cirrhosis follows when masses of liver die. There are many causes of cirrhoses, but often the exact cause is unknown. Cirrhosis leads to liver failure. It also leads to obstruction to the flow of blood from the portal vein.

\section{Design of Fuzzy Controller:}

the fuzzy controller takes the input as the symptoms fever, loss of appetite, nausea, jaundice, dark urine, Stomach pain, Vomiting, Dull aches , Changes in liver, Failing health, Loss of weight, Headache and depending upon their membership values the fuzzy controller will generate the disease as output Hepatitis A, Hepatitis B, Cirrhosis. On the basis of the symptoms another fuzzy has been created.

This controller will take the symptom as input variable. These symptoms are the listed above. Depending upon the degree of truthiness of these input variables the diseases can be asserted. It is observed that changes in one symptom will change the diseases depend upon the truthiness value. If the value of the symptom jaundices high the chance of occurring the disease Hepatitis B is more than the hepatitis A. the fuzzy controller takes the 12 inputs and produce the 3 outputs. For example the variable changes in liver can be divided into three categories Mild changes, Moderate Changes, Severe Changes. We define the range for individual variable from 0-10. If the variable value is from $0-4$, the variable is grouped in 'mild changes' in the liver, if it is from $4-7$, variable 
grouped into Moderate changes. If it ranges from $7-10$, the variable grouped into severe changes. Each variable is combination of the triangular and trapezoidal membership function. The modifiers Mild and Severe linguistic variable represented as trapezoidal membership functions. It has truthiness value from $0-1$. The modifier Moderate represented as the triangular membership function. Show the example of liver system.

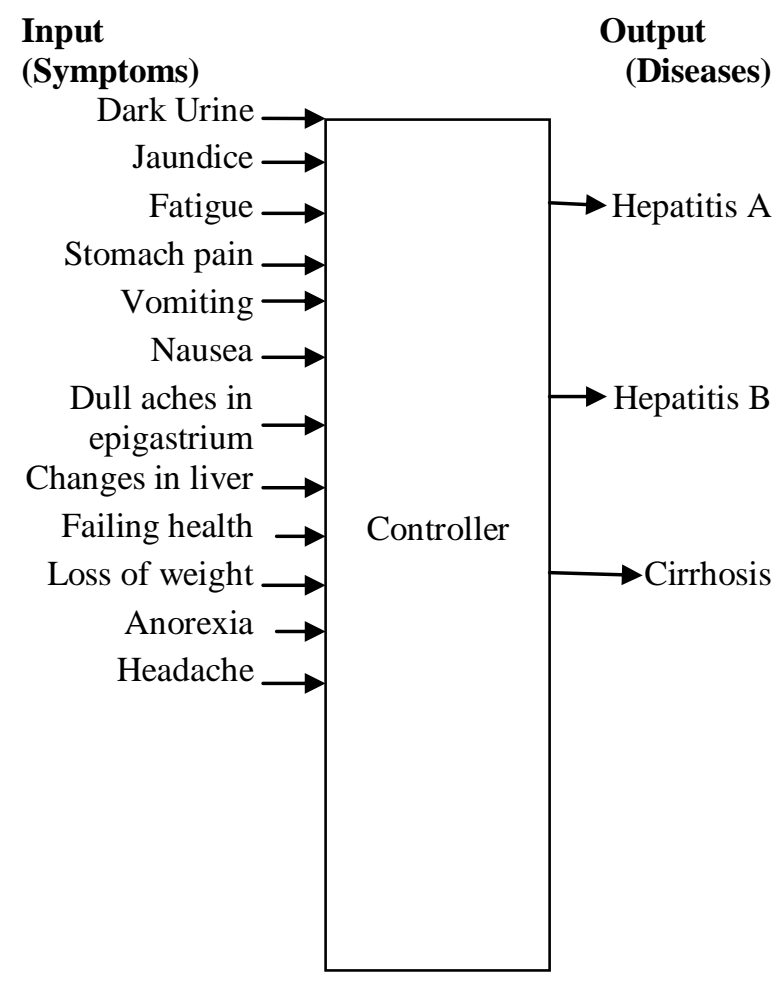

Figure 2 Diseases of liver

Algorithm

\section{Inputs: - Age, Gender and some vital sign and symptoms.}

2. Output:- Diagnose the particular Disease and suggest the specialist

3. Input variable assigned with fuzzy variable. And has the membership function.

4. Rules are fired on the basis of the membership function.

5. Finally disease is confirmed on the basis of the weight age of input variable. And corresponding

This system is developed using the mat lab. The overall classification is done using the fuzzy logic toolbox. The GUI provides the communication between the user and system. GUI also called the graphical display in one or two windows containing the different parts called components. The components contain the push button, static text, edit text, popup menu, slider etc. Graphical user interface showing the vital sign and symptoms which appears. In this GUI the patient have to fill the range of symptoms according to the mild, 
moderate, and severe. After clicking on the button, the system will tell the disease whether it is present or not. If the disease is present then it will tell to the patient whether it is on first stage, second stage or third stage. And at the end it tells the corresponding specialist for patient's treatments. Inference engine where fuzzified value is defuzzified in the model for decision making. The symptoms of the patient are fed into the system. And the based on the weights of the symptoms store into the database. After adding the symptoms the value is fuzzified. The structure of knowledge is combination of facts and rules of the diseases related to different part of human body.

In above GUI the patient fill the range of symptoms pain in ear is moderate (6), redness in ear is high (9),

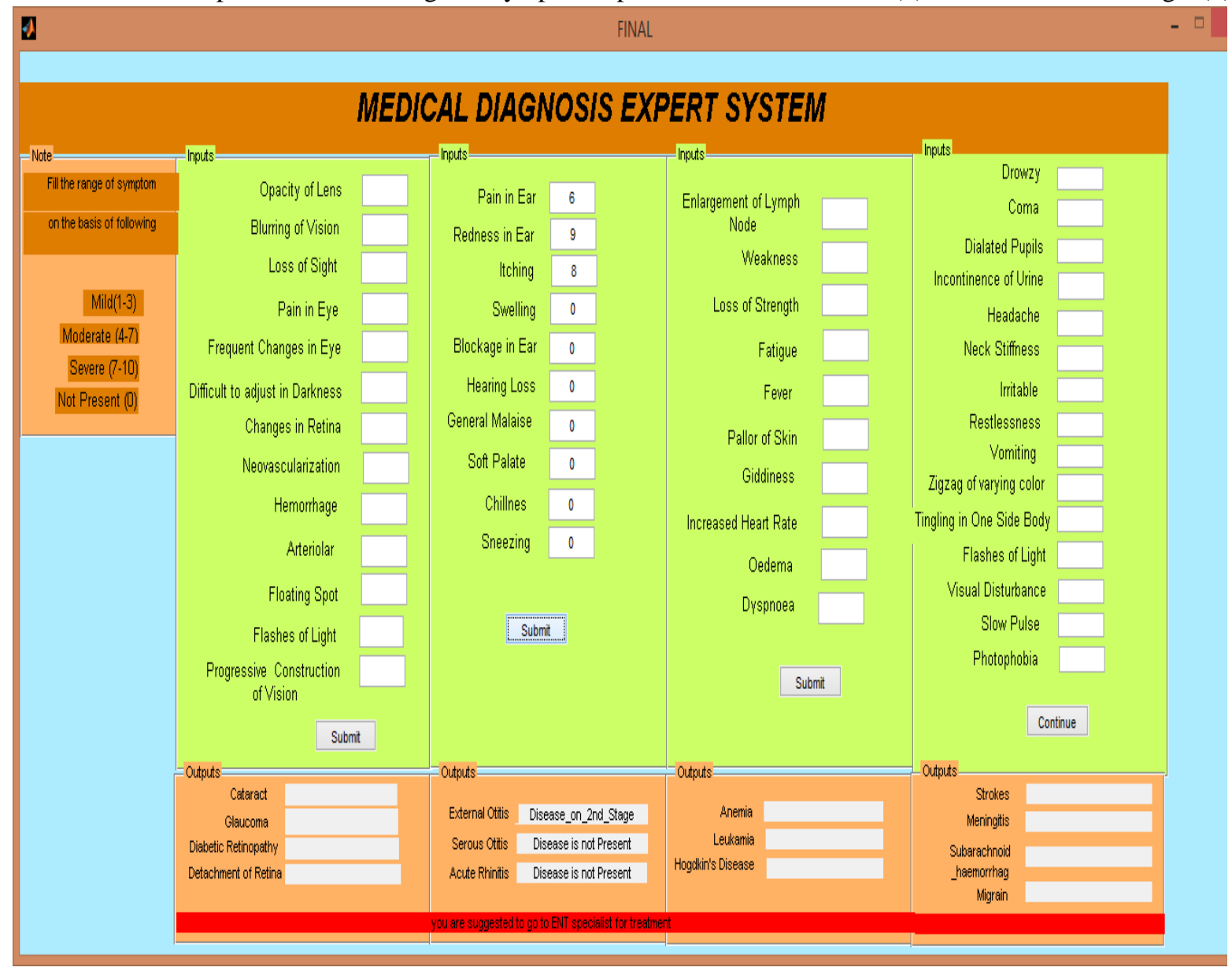

Figure 3 Medical Diagnosis Expert System

itching in ear is also high (8), the system tells the corresponding disease i.e. External otitis and also gives the stage of particular disease i.e. on the $2^{\text {nd }}$ Stage. At last it suggests the expert that is ENT Specialist for their treatments. It saves the time of the patient as well as the doctor or physician.

\section{COCLUSION}

we describe the fuzzy logic based medical expert system for diagnosing of the disease related to disorder of liver, disorder of blood, disorder to eye, disorder to kidney, disorder to ear nose throat, disorder of nervous system. We know there are many applications which are build on fuzzy data analysis but those which are build on current database are rare. This system can be used by the doctors, physician in our daily life. The proposed medical diagnosis expert system, one can allow the physician to follow the same process to diagnose the diseases and he/she will able to suggest the specialist in easy way. A lot of research has been done in medical field but more research increase the accuracy of the system. So many properties of this system remain to investigate. Future application for the database should be good. Now a day's most of the modern hospitals use the computer based records than paper based. Now it would be more easily to acquisition the data from records for the machine diagnosis. An expert should evaluate the quality performance of this system. This expert system succeeded in estimating the diseases of liver, diseases of eye, diseases of kidney, diseases of nerve. There are 35 cases in which system perform perfect out of 45 cases. The future work, the more diseases will be added to the system and it will be tested. 


\section{REFERENCES}

[1] H.S.Hota, "Diagnosis of Breast Cancer Using Intelligent Techniques", International Journal of Emerging Science and Engineering (IJESE) ISSN: 2319-6378, Volume-1, Issue-3, page no. 45 - 58 January 2013

[2] D. G. Bounds, P. J. Lloyd, B. Matthew, and G. Waddell, "A Multi Layer Perceptron Network for the Diagnosis of Low Back Pain", International Conference on Neural Networks, San Diego, CA, volume2, page no. 58-64, 2012

[3] Priynka Sharma, "Decision support system for Malaria and Dengue", International journal of information and computation technology, ISSN: 0974-2239 Volume 3, page no. 76-82, 2013

[4] S.Krishan Anand, "Design and implementation of fuzzy expert for estimating the level of Asthma", Middle-East Journal of Scientific Research Volume -2 page no. 51-59, 2013.

[5] Mir Anamul Hasan, Md. Sher-E-Alam and Ahsan Raja Chowdhury," Human Disease Diagnosis Using a Fuzzy Expert System”, Journal of Computing, Volume 2, ISSN2151-9617, page no. 81-90, JUNE 2010

[6] E.P.Ephzibah, "A Neuro Fuzzy Expert System For Heart Disease", An International Journal of computer science (CSEIJ), Volume 2, ISSN: 14351444, February 2012

[7] Ahmad A. Al-Hajji," New Expert System for Pediatric Respiratory Diseases by Using Neural Networks", Internet conference of computer and IT(ICCIT ),2012

[8] Y. H. Kimbi,“A medical diagnostic support system for the management of Hypertension (meddiag)”, International Journal of Medical and Biosciences Volume 3, page no. 41-55, March 2011

[9] Yanqing Duanb ,Fish-Expert," A web based expert system for fish disease diagnosis", Expert Systems with Applications, An International Journal of computer science (CSEIJ) 23, 311-320, 2002

[10] A K Verma \& K Seetharam, "An expert diagnostic tool for engineering systems", (journal of scientific \& Industrial research, vol 53, pp 601-603, 2002

[11] Sey Shamsadin Athari, "Immune response Systems with Applications", Middle-East Journal of Scientific Research 13 (4): ISSN 1990-9233, IDOSI Publications, pp: 489-498, , 2013 